\title{
Cellular Positioning Using Fingerprinting Based on Observed Time Differences
}

\author{
David Gundlegård, Awais Akram, Scott Fowler and Hamad Ahmad \\ Mobile Telecommunications \\ Department of Science and Technology \\ Linköping University Norrköping, Sweden
}

\begin{abstract}
Cellular positioning has been a very active research area for the last decade. Large improvement in accuracy has been made to support, for example, e-call and other location-based services. Traditionally, cellular positioning has been limited to cellular operators equipped with expensive synchronization hardware in order to achieve good accuracy. Lately, third parties have employed fingerprinting methods to enable positioning systems independent from the cellular operators. With improved available processing power, denser cellular networks, cheaper data collection and efficient pattern matching algorithms, the fingerprinting positioning methods have also gained popularity. In this paper, we analyzed the potential of using System Frame Number (SFN) - SFN observed time differences, which are traditionally used in Time Difference of Arrival (TDOA) positioning, for fingerprinting-based positioning. A field test was performed using measurements from TEMS (Telecommunication Management System) Investigation. By combining SFN-SFN observed time differences with Received Signal Code Power (RSCP) measurements, we demonstrated an improved accuracy of the fingerprinting method by $20 \%$ compared to only using RSCP measurements. The results are promising and show good potential in using SFN-SFN observed time differences for positioning based on fingerprinting.
\end{abstract}

\section{INTRODUCTION}

With the rapid growth of wireless systems, there is a huge demand for accurate and effective positioning methods for indoor as well as outdoor. Recently, cellular positioning has gain attention with its improved accuracy. Despite the rise of Global Positioning System (GPS) technologies, cellular positioning remains to be significant because it can supplement GPS with the following features: Improved urban canyon coverage, reduced time-to-first-fix (TTFF), less battery drain, positioning support for non-GPS terminals, GPS assistance data and Indoor coverage. Furthermore, there are distinctive features of cellular positioning that are missing in GPS, such as personal integrity aspects and potential for the use of cell phone signalling data. Inevitably, location data almost always include an important personal integrity component and the use of cellular positioning can be much less intrusive to personal integrity comparing to GPS positioning. This is due to the type of measurements available in legacy cell phones, e.g. Received Signal Strength (RSS) measurements or rough propagation times. These type of measurements reduces the spatial resolution of the positioning system such that it is no longer possible to extract information associated with personal integrity, such as the exact address that the cell phone has visited. Such information is likely to be revealed with the use of GPS.

Today, nearly $100 \%$ cell phone penetration rate with extensive cell phone use is found in many countries. As a result, the location data generated in cellular networks has gained attention as it has the potential for a large number of applications. For example, cellular network data can be used for urban planning, evacuation management, and road traffic information. Certain types of road traffic information, e.g. travel time and traffic flow estimation, require sub cell location accuracy. In this paper we propose to use existing signaling messages with observed time difference measurements to improve location accuracy whenever these measurements are available, e.g. during the soft handover process. By using a fingerprinting methodology with our proposed method will result in an more accurate positioning without the need to install expensive hardware to support synchronization.

\section{RELATED WORK}

Cell phone positioning has gained intensive research interest especially since the Federal Communications Commission (FCC) requirements on cell phone location accuracy for emergency calls was released in 2001 [2]. Due to denser network deployments, increased availability of non-operator controlled positioning and improved positioning methods; more work has recently been focusing on RSS-based positioning. In [4], fundamental limits of cellular positioning were analyzed with special focus on RSSbased fingerprinting techniques. A good overview of fundamental limits of cellular positioning systems, including map matching and motion models was given in [8].

In [1], simulation were performed in order to measure the position accuracy for Time of Arrival (TOA) in WCDMA. It was identified that when 300 random User Equipment (UE) positions were selected, the 67\% Circular Error Probability (CEP-67) is $125 \mathrm{~m}$ for urban environments and $50 \mathrm{~m}$ for suburban environments. The positioning accuracy of suburban environments was better due to less multipath propagation.

Simulation of TDOA was performed in [7] for WCDMA and CDMA2000. The results show that the accuracy is affected severely by the multipath conditions, the integration time and whether idle periods are used by the base stations. The CEP-67 was approximately $100 \mathrm{~m}$ for the urban environments. Angle of Arrival (AOA) and TDOA were evaluated for WCDMA in [3], 
and it was noted that the root mean square error (RMSE) in meter was lower in TDOA compare to AOA with constant Signal to Noise Ratio (SNR). The RMSE was $100 \mathrm{~m}$ for TDOA and $125 \mathrm{~m}$ for AOA.

Fingerprinting (also known as Pattern Matching or Database Correlation Method (DCM)) is a commonly used method for positioning because it has the ability to accommodate legacy terminals. In this method, a training database is created using an offline measurement phase. In the online phase the measurements collected are compared with the training data to find the best matching fingerprint in the database, which then serves as the position estimate.

Fingerprinting uses measurements made by the Base Station, the UE, or a combination of both, and these measurements are then compared with the already stored fingerprints to estimate the position. There are various measurements which can be used to create the database, but a commonly used measurement is Received Signal Code Power (RSCP). In [10], two matching algorithms were used, where the distance was calculated using Least Mean Square (LMS) and an Exponential (EXP) function. The EXP function performed best and the CEP-67 was $83 \mathrm{~m}$ for urban environments and $607 \mathrm{~m}$ for rural environments. In [9] an efficient way of building the fingerprint database is shown, where reference measurements are located using observed time difference of arrival (OTDOA) in Long Term Evolution (LTE) networks.

However, none of the previous work considered using fingerprinting based on observed time differences. Thus, in this paper we focus on using existing Observed Time Difference measurements, and these measurements are further described in section III.

\section{LOCATION DATA}

The accuracy of the location estimate is based on the available measurements, which depends on which state the terminal is in. This paper will focus on network-based positioning in which the UE sends measurement reports to the network and subsequently, the network estimates the position of the device. Our approach mainly used information based on Layer 3 messages at uplink $\mathrm{DCCH}(\mathrm{UL}-\mathrm{DCCH})$.

When an user is moving in active mode, it needs to remain connected and the network continuously needs measurement reports from the UE. By doing this, the network will accurately help the UE. These measurement reports are messages in the RRC protocol which contains information of the current active set and monitored neighbours with specification as given below [6]:

- $\mathrm{OFF}=$ Number of frames with a range of 0 to 255

- $\mathrm{TRxSFN}_{j}=$ Time of received neighboring $\mathrm{P}-\mathrm{CCPCH}$ from cell $\mathrm{j}$

- $\mathrm{TRxSFN}_{i}=$ Time of received serving $\mathrm{P}-\mathrm{CCPCH}$ from cell $\mathrm{i}$

- $\mathrm{Tm}=\mathrm{TRxSFN}_{i}-\mathrm{TRxSFN}_{j}$

- $\mathrm{SC}=$ Scrambling Code ID

- $\quad$ Ec-NO in $\mathrm{dB}$

- $\quad$ RSCP in $\mathrm{dBm}$

- $\quad$ SFN-SFN OTD

The physical layer uses system frame number (SFN), while SFN-SFN represents the difference SFNs of two Node Bs ${ }^{1}$; one as active and the other as monitored neighbour. Using information of Tm and OFF, the SFN-SFN Obeserved Time Difference (OTD) can be calculated in chips as [1]:

$$
S F N-S F N=O F F \cdot 38400+T m
$$

where $T m=T R x S F N_{j}-T R x S F N_{i}$, in chip units, range $(0,1, \ldots 38399)$ chips. $T R x S F N_{j}$ stands for the beginning time of a received neighbouring P-CCPCH from cell ${ }_{j}$ and $T R x S F N_{i}$ represents the beginning time of P-CCPCH frame from serving cell ${ }_{i}$ OFF $=$ $\left(S F N_{i}-S F N_{j}\right)$, given in number of frames $(0,1, \ldots, 255)$.

This SFN-SFN value is measured and transmitted to the network when the terminal is in state of soft handover. We used this SFN-SFN value as a fingerprint to estimate the UE location.

\footnotetext{
${ }^{1}$ In this paper Node B is the Base Station (BS)
} 


\section{Methodology}

In our methodology of fingerprinting we used RSCP and SFN-SFN observed time difference on the network side to estimate the position. By doing this there is no need to install any additional equipment on the network side. This makes it possible to estimate the location of legacy terminals based on the measurement reports transmitted in active mode. The main focus of this paper is to explore the potential of SFN-SFN OTD for positioning using the fingerprinting method and compare the results to standard fingerprinting based on RSCP. For further improvement, the SFN-SFN and RSCP measurements were combined.

Using Layer 3 messages from TEMS Investigation, SFN-SFN OTD was calculated for different scrambling codes. In the first phase, a database was created for the collection of the data by using SFN-SFN OTD between SFN of Active Set (AS) and Monitoring Neighbours (MN). In the second phase, the UE was located by matching its current SFN-SFN OTD with the fingerprint stored in the database.

\section{DATA COLlection} 1.

The area around Linköping University, Campus Norrköping was chosen for the drive test measurements as shown in Figure

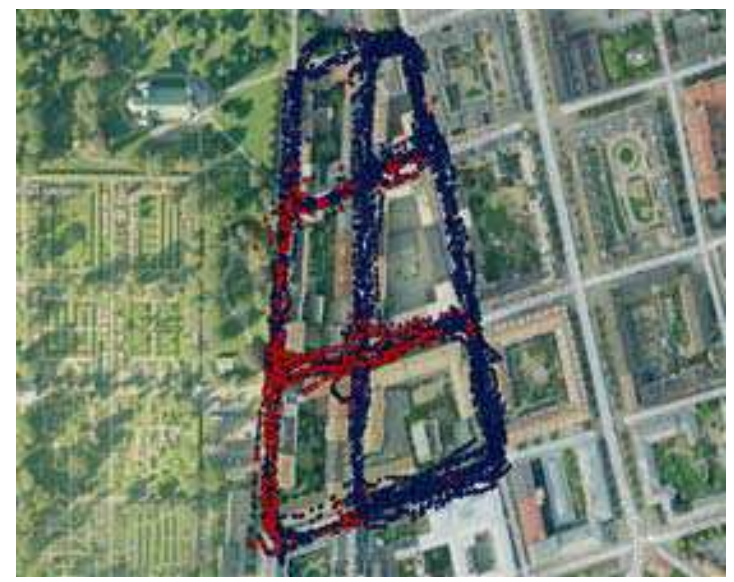

Figure 1: Drive Test Area with RSCP measurements in blue and SFN-SFN OTD measurements in red.

The area of the drive test was $315 \mathrm{~m} \times 215 \mathrm{~m}$ with a maximum diagonal distance of $375 \mathrm{~m}$. This area is considered as urban and mostly covered with buildings and trees. We were able to identify 12 different Node Bs, all with different coverage and signal strength characteristics. During the drive test, a GPS receiver was connected with a Laptop where TEMS Investigation stored all measurements.

RSCP, Ec-N0, time, location can be extracted directly by TEMS Investigation 9.0, but SFN-SFN observed time difference needs to be extracted from L3 messages which are saved as text files. L3 messages contain the value of OFF and Tm against each SC. MATLAB was used to extract these values from the L3 messages to calculate SFN-SFN OTD according to Equation (1).

To design the algorithm it is important to know the behaviour of different Node Bs with respect to RSCP and SFN-SFN OTD in test drive area. For this purpose, patterns of RSCP and SFN-SFN OTD were studied in detail.

The drive test area shown in Figure 2 describes different active sets at different location which are distinguished by colors. The color schemes show that the change of color occurs when soft handover is taken place. Mostly SC 292 is acting as $A S$ because of its strong RSCP and Ec-NO.

Figure 3 shows the SFN-SFN OTD measurements in a map where each color represents different pairs of Node Bs. SFN-SFN OTD is only available when a soft handover takes place and SFN-SFN OTDs are calculated for all pair of Node Bs that are identified.

SFN-SFN OTD varies with space and the Node Bs report the value of SFN after every 20ms to the UE via BCH [5]. The data for SFN-SFN OTD in the test drive area becomes dense since several test drives are performed.

The method used to find the position of the UE is Euclidean Distance, in which the reported measurements from the UE are compared with the stored fingerprints in the database. The location with smallest Euclidean Distance is considered as the estimated position of the UE. Both RSCP and SFN-SFN OTD measurements are used to estimate the position. Finally, the accuracy is evaluated when using both measurements simultaneously.

The Euclidean distance between measurement and fingerprint is calculated as follows: 


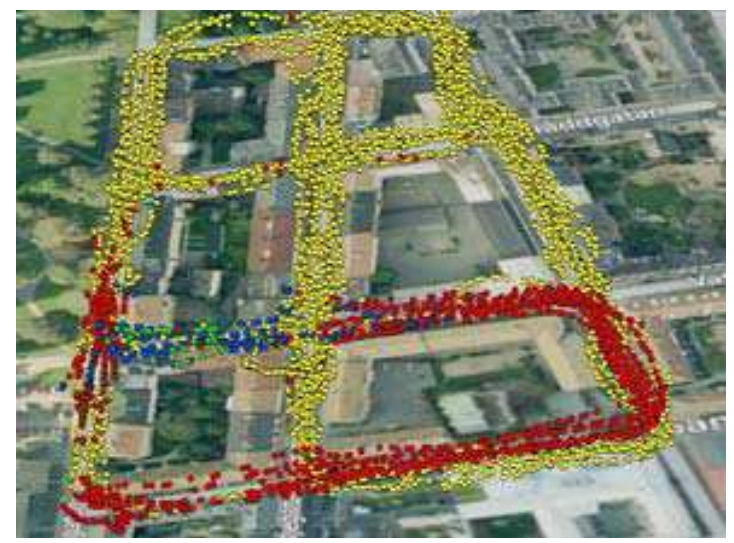

Figure 2: RSCP of different Node Bs. Node B with SC 292 mainly serves as active set which is in yellow, while SC 346, SC 126, SC 372, SC 204 are shown in red, brown, green and blue, respectively.

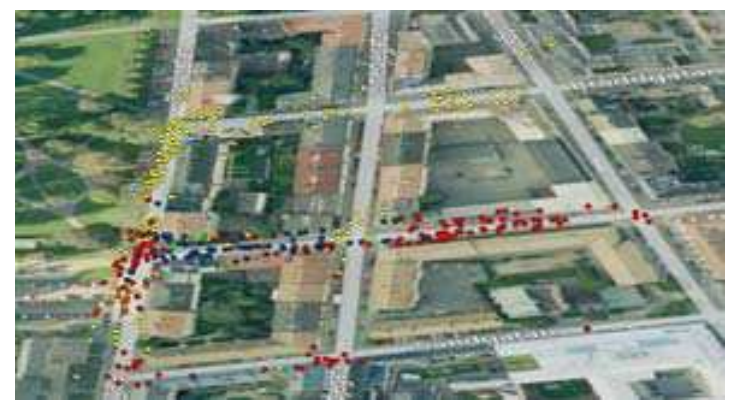

Figure 3: SFN-SFN at soft handover location. SC 292 is shown in yellow, while SC 346, SC 126, SC 372, SC 204 are shown in red, brown, green and blue, respectively.

$$
d_{k}^{E D}=\sqrt{\sum_{i \in M}\left(f_{k i}-m_{i}\right)^{2}}
$$

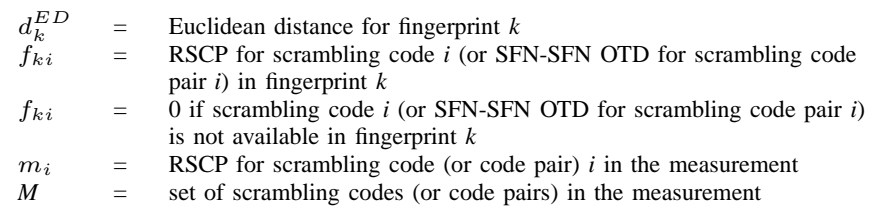

\section{RESULTS}

According to Figure 1, the blue color shows the whole area where measurements are collected, which has the dimensions of $315 \times 215 \mathrm{~m}$ with maximum diagonal distance of $375 \mathrm{~m}$. The results for this area are compared with the FCC requirements to analyze whether the results fulfil the required standard. The FCC requirement for network based positioning is that the location error must be below $100 \mathrm{~m}$ for $67 \%$ of the time and must be below $300 \mathrm{~m}$ for $95 \%$ of the time. Due to the small size of the area the results should be seen as a lower bound of the error estimates.

The algorithm was evaluated for two different scenarios, 1) was for the complete area in which the estimated accuracy was analyzed only by considering RSCP, and 2) the other scenario was for soft handover area in which the estimated accuracy was analyzed for both RSCP and SFN-SFN OTD. Also, we did a hybrid technique for this scenario.

The results for the complete area and soft handover area were analyzed with three different cases:

Case 1: Average of 3 best matching fingerprints (FP)

Case 2: Average of 5 best matching FP

Case 3: Average of 7 best matching FP 


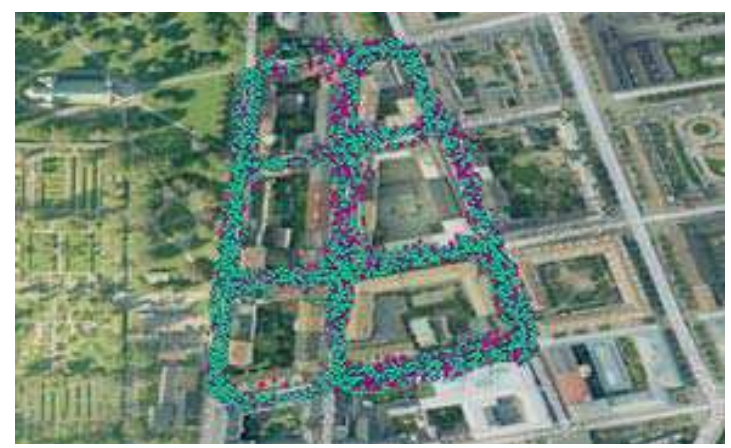

Figure 4: Complete Area with 67\% Fingerprints (pink color) and 33\% of Evaluation Measurements (green color).

Table I: Location Error of RSCP for Complete Area.

\begin{tabular}{|c|c|c|c|}
\hline Cumulative & \multicolumn{3}{|c|}{ Location Error (m) } \\
\cline { 2 - 4 } Distributive & Case 1 & Case 2 & Case 3 \\
\cline { 2 - 4 } Function (\%) & Avg. of top 3 FP & Avg. of top 5 FP & Avg. of top 7 FP \\
\hline 67 & 46.00 & 45.52 & 44.06 \\
\hline 95 & 211.35 & 210.87 & 200.98 \\
\hline
\end{tabular}

The results for the complete area is shown in Table I. For CEP-67 the difference was very small between the different cases. However, for the CEP-95 metric case 3 had a $10 \mathrm{~m}$ improvement of the estimated accuracy compared to the other two cases. The CEP-95 being better when we use more fingerprints in the estimate is natural since large errors are typically removed. The CEP-67 being better, although it was a small difference, can indicate that we had relatively noisy measurements. The maximum error for the estimated position was $268 \mathrm{~m}, 260 \mathrm{~m}$ and $252 \mathrm{~m}$ for the case 1, 2 and 3, respectively.

The dimensions of soft handover area are $220 \times 215 \mathrm{~m}$ with maximum diagonal distance of $305 \mathrm{~m}$. In this scenario, three methods were evaluated, using RSCP measurements, SFN-SFN OTD, or the hybrid of the two. To analyze and compare RSCP, SFN-SFN OTD and hybrid approaches the size of the area should be the same, which means that we evaluate RSCP, SFN-SFN OTD and hybrid also for the soft handover area. The database was divided into two parts by taking $2 / 3$ of the measurements as fingerprint samples and 1/3 as evaluation samples, which is shown in Figure 5. The results for soft handover area for all three evaluation methods were analyzed using the same three cases as discussed earlier.

The results for the three cases of RSCP in soft handover area were compared in Table II, here we see that case 1 was performing best for CEP-67, while case 3 was performing well and showed significant better result for CEP-95 and case 2 was somewhere in between the case 1 and 3 in respect of performance. It seems logical that the error is smoothed using a combination of more fingerprints as estimate. The maximum error for the estimated position was $257 \mathrm{~m}$.

In Table III we see that for SFN-SFN OTD Case 2 is performing better in both CEP-67 and CEP-95. This is most likely because the optimal number of FP to average over in SFN-SFN OTD is 5, whereas with more FP the results get worse for both CEP-67 and CEP-95. In case of RSCP, this optimal value is 7 FP and if it is increased from 7 the results become worse. The maximum error for the estimated position is $230 \mathrm{~m}$.

In Hybrid technique both RSCP and SFN-SFN OTD are used at the same time. The results of the three cases for the hybrid

Table II: Location Error of RSCP for Handover Area

\begin{tabular}{|c|c|c|c|}
\hline Cumulative & \multicolumn{3}{|c|}{ Location Error (m) } \\
\cline { 2 - 4 } Distributive & Case 1 & Case 2 & Case 3 \\
\cline { 2 - 4 } Function (\%) & Avg. of top 3 FP & Avg. of top 5 FP & Avg. of top 7 FP \\
\hline 67 & 26.16 & 30.84 & 33.91 \\
\hline 95 & 72.44 & 64.63 & 61.69 \\
\hline
\end{tabular}

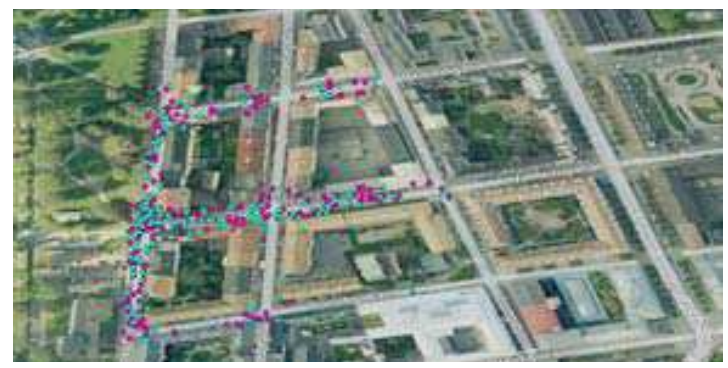

Figure 5: Handover Area with 67\% Fingerprints (pink color) and 33\% of Online Data (green color). 
technique are shown in Table IV. From Table IV, it shows better results as compared to RSCP. The RSCP, SFN-SFN OTD and hybrid technique are compared in Figure 5. It was noticed that for all the three methods, the location error was below $100 \mathrm{~m}$ for CEP-67 and below $300 \mathrm{~m}$ for CEP-95.

For the hybrid method there was very little difference between the three cases in terms of CEP-67 performance. However, for CEP-95 case 3 performs significantly better than the other two cases where it has an error of only $55 \mathrm{~m}$ and case 1 and case 2 has error of $71 \mathrm{~m}$ and $66 \mathrm{~m}$, respectively. The maximum error is $222 \mathrm{~m}$. The hybrid technique is showing better result because we have access to more data and we managed to combine it efficiently. The Euclidian distance is calculated for all measurements simultaneously, instead of just weighting the results of the RSCP and SFN-SFN OTD methods.

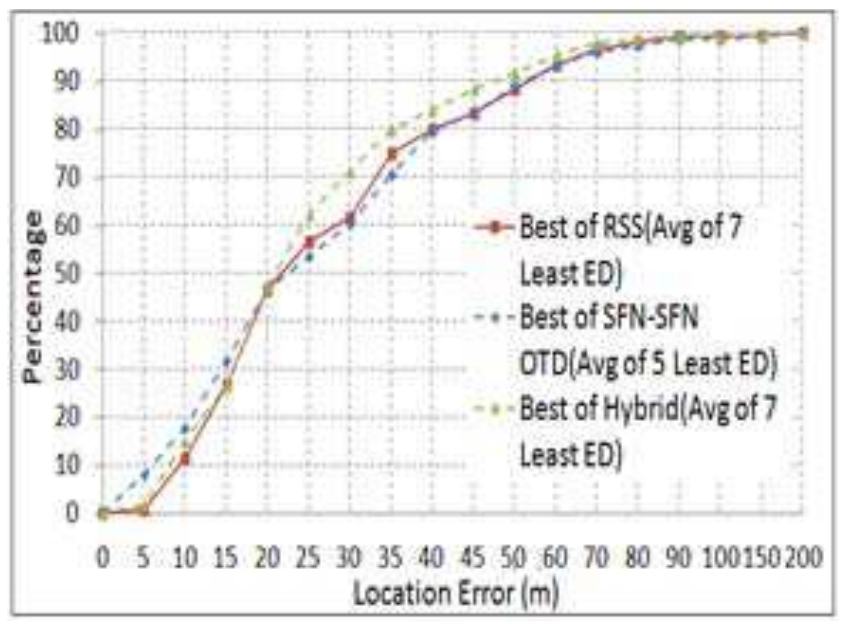

Figure 6: CDF Comparison of RSCP, SFN-SFN OTD and Hybrid.

The results for RSCP, SFN-SFN OTD and Hybrid techniques are analyzed with three different cases, i.e. average of top 3 FP, average of top 5 FP and average of top 7 FP. It is noticed that in case of RSCP, it gives better estimated position if average of top $7 \mathrm{FP}$ are considered and in case of SFN-SFN OTD, results are better with average of 5 top FP while Hybrid technique performs significantly better for average of top 7 FP. To analyze the performance of RSCP, SFN-SFN OTD and Hybrid techniques, their results are compared with each other as shown in Table $\mathrm{V}$ and Figure 6. By comparing the results of these three techniques it is concluded that SFN-SFN OTD is performing approximately same as RSCP but the Hybrid technique shows significant improvement in accuracy, especially for higher confidence levels.

From Table V we see that the SFN-SFN OTD measurements are performing very similar to regular RSCP measurements when used for fingerprinting. However, by combining the measurements we are able to reduce the location error significantly. This means that the measurements are uncorrelated enough to improve the location estimate when used in a Euclidian distance nearest neighbour matching algorithm.

\section{CONCLUSION}

We concluded that the fingerprinting approach based on standardized SFN-SFN OTD measurements is possible to use to locate legacy terminals when the measurements are available, for example during soft handover. Comparing results of RSCP, SFN-SFN OTD and Hybrid approach it is concluded that SFN-SFN OTD shows similar results as for RSCP measurements, whereas the hybrid approach improves the location accuracy. The hybrid approach improves the location accuracy with $20 \%$ compared to only using RSCP measurements, which is promising when we want to improve the location accuracy for legacy terminals in specific areas

\section{ACKNOWLEDGMENT}

Scott Fowler was partially supported by EC-FP7 Marie Curie CIG grant, Proposal number: 294182.

Table III: Location Error of SFN-SFN OTD for Handover Area

\begin{tabular}{|c|c|c|c|}
\hline Cumulative & \multicolumn{3}{|c|}{ Location Error (m) } \\
\cline { 2 - 4 } Distributive & Case 1 & Case 2 & Case 3 \\
\cline { 2 - 4 } Function (\%) & Avg. of top 3 FP & Avg. of top 5 FP & Avg. of top 7 FP \\
\hline 67 & 31.67 & 33.29 & 33.82 \\
\hline 95 & 68.28 & 61.22 & 68.99 \\
\hline
\end{tabular}

Table IV: Location Error of Hybrid for Handover Area

\begin{tabular}{|c|c|c|c|}
\hline Cumulative & \multicolumn{3}{|c|}{ Location Error (m) } \\
\cline { 2 - 4 } Distributive & Case 1 & Case 2 & Case 3 \\
\cline { 2 - 4 } Function (\%) & Avg. of top 3 FP & Avg. of top 5 FP & Avg. of top 7 FP \\
\hline 67 & 26.97 & 25.29 & 26.81 \\
\hline 95 & 70.78 & 65.75 & 55.62 \\
\hline
\end{tabular}


Table V: Location Error of RSCP, SFN-SFN OTD and Hybrid approach for the Handover Area

\begin{tabular}{|c|c|c|c|}
\hline Cumulative & \multicolumn{3}{|c|}{ Location Error (m) } \\
\cline { 2 - 4 } $\begin{array}{c}\text { Distributive } \\
\text { Function } \\
(\%)\end{array}$ & Case 1 & Case 2 & Case 3 \\
\cline { 2 - 4 } & $\begin{array}{c}\text { RSCP } \\
\text { (Avg. of top 3 FP) }\end{array}$ & $\begin{array}{c}\text { SFN-SFN OTD } \\
\text { (Avg. of top 5 FP) }\end{array}$ & $\begin{array}{c}\text { Hybrid } \\
\text { (Avg. of top 7 FP) }\end{array}$ \\
\hline 67 & 33.91 & 33.29 & 26.81 \\
\hline 95 & 61.69 & 61.22 & 55.62 \\
\hline
\end{tabular}

\section{REFERENCES}

[1] M. Cedervall. Mobile positioning for third generation wcdma systems. In Universal Personal Communications, 1998. ICUPC '98. IEEE 1998 International Conference on, volume 2, pages 1373-1377 vol.2, 1998.

[2] F. Gustafsson and F. Gunnarsson. Mobile positioning using wireless networks: possibilities and fundamental limitations based on available wireless network measurements. Signal Processing Magazine, IEEE, 22(4):41-53, 2005.

[3] M. Nur-A-Alam and M.M. Haque. A least square approach for tdoa/aoa wireless location in wcdma system. In Computer and Information Technology, 2008. ICCIT 2008. 11th International Conference on, pages 686-690, 2008.

[4] M. Pettersen, R. Eckhoff, P.H. Lehne, T.A. Worren, and E. Melby. An experimental evaluation of network-based methods for mobile station positioning. In Personal, Indoor and Mobile Radio Communications, 2002. The 13th IEEE International Symposium on, volume 5, pages 2287-2291 vol.5, 2002.

[5] Shing-Fong Su. The umts air-interface in rf engineering: Design and operation of umts networks. McGraw Hill Professional ISBN: 0-07-148866-9, 2007.

[6] 25. 3GPP TS 25.215 v10.0.0. Technical Specification Group Radio Access Network; Physical layer; Measurements (FDD).

[7] S.S. Wang, M. Green, and M. Malkawi. Analysis of downlink location methods for wcdma and cdma2000. In Vehicular Technology Conference, 2001. VTC 2001 Spring. IEEE VTS 53rd, volume 4, pages 2580-2584 vol.4, 2001.

[8] T. Wigren. Adaptive enhanced cell-id fingerprinting localization by clustering of precise position measurements. Vehicular Technology, IEEE Transactions on, 56(5):3199-3209, 2007.

[9] T. Wigren, A. Kangas, Y. Jading, I. Siomina, and C. Tidestav. Enhanced wcdma fingerprinting localization using otdoa positioning measurements from lte. In Vehicular Technology Conference (VTC Fall), 2012 IEEE, pages 1-5, 2012.

[10] D. Zimmermann, J. Baumann, M. Layh, F. Landstorfer, R. Hoppe, and G. Wolfle. Database correlation for positioning of mobile terminals in cellular networks using wave propagation models. In Vehicular Technology Conference, 2004. VTC2004-Fall. 2004 IEEE 60th, volume 7, pages 4682-4686 Vol. 7, 2004.

\section{APPENDIX - LIST OF ACRONYMS}

AOA Angle of Arrival

AS Active Set

CEP Circular Error Probability

EXP Exponential

FCC Federal Communications Commission

GPS Global Positioning System

LMS Least Mean Square

MN Monitoring Neighbours

OTD Observed Time Difference

RMSE Root Mean Square Error

RSCP Received Signal Code Power

RSS Received Signal Strength

SFN System Frame Number

SNR Signal to Noise Ratio

TDOA Time Difference Of Arrival

TEMS Telecommunication Management System

TOA Time of Arrival

TTFF Reduced Time-to-First-Fix

UE User Equipment 